\title{
Research and Magnetic Field Simulation of Automotive Magneto-rheological Fluid Dual-clutch Transmission
}

\author{
Demin Chen ${ }^{1, a}$, Hong Zhang ${ }^{1, b}$ and Qingge Cai ${ }^{2}$ \\ ${ }^{1}$ Academy of Armored Force Engineering, Beijing, China \\ ${ }^{2}$ Unit 63981 of PLA, China \\ admchn@163.com, bbitzhanghong@foxmail.com
}

Keywords: Magneto-rheological fluid; dual clutch; magnetic field; simulation.

\begin{abstract}
Based on the magneto-rheological effect of magneto-rheological fluid (MRF) and the work characters of dual-clutch transmission (DCT) put forward a new type of automobile transmission: magneto-rheological fluid dual clutch transmission (MRDCT). The MRDCT has simple structure, no wear, low energy consumption and good controllability. The work principle of magneto-rheological dual clutch (MRDC) is expounded and the torque calculation formulas are established. A finite element model of the MRDC is established in Ansoft to simulate the magnetic field distribution. The results show that the two clutches have no electromagnetic interference.
\end{abstract}

\section{Introduction}

Intellectualization is the development direction of automobile, and also an evolution stage of automobile industry. It includes the electrification of the vehicles and the development of the internet of vehicles. The electrification of the actuators of the vehicles is an important part of that. The clutch plays a very important role in the transmission system. As an intelligent material, MRF has been widely applied in industry [1,2,3], MRF clutch (MRC) is one of them $[4,5]$.

In the field of automobile transmission, DCT got fast development since the end of last century and has been applied on many cars. But the structures of the dual clutch and the control valve are complex and have high requirements on machining accuracy.

In this paper we put forward a new type of automobile transmission: MRDCT, which combined the magneto-rheological effect MRF and the work characters of DCT.

\section{The MRC}

The MRF. MRF is a kind of intelligent material, and it was first invented by Rabinow in 1948. Normally, the MRF contains the magnetic particles and the base fluid and other additives.

Without magnetic field, the magnetic particles of MRF float in the base fluid randomly. While under the magnetic field, the particles can shift into Bingham plastic solid, and form chain-structure within millisecond level. The two different shapes of MRF are shown in figure 1 . The left is without magnetic field.
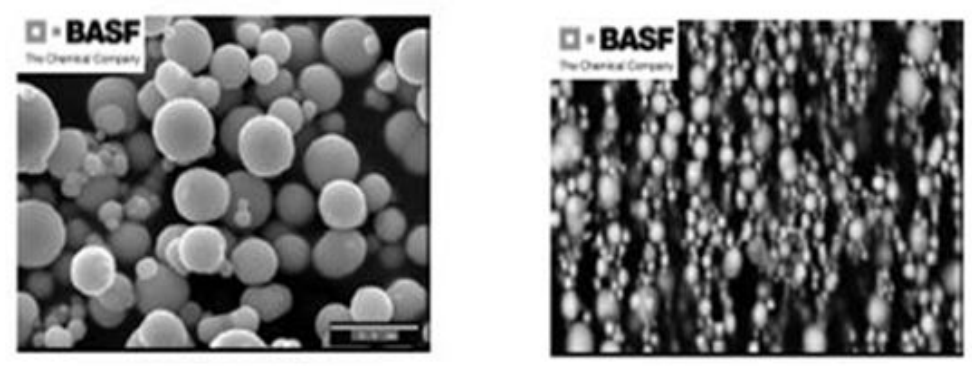

Fig.1 The two shapes of the MRF 
This effect is called magneto-rheological effect. The shear yield stress of the MRF will change along with the magnetic flux density [6].

The MRF has the characteristics of reversible, fast reaction, easily controlled and no wear. It is widely used in the electrical control system and mechanical system which ask for a simple structure, quiet work environment and rapid response.

Limited by the technology, the study of the MRF developed slowly at the beginning. In the last two decades, with the development of material science and the progress of the manufacturing, the problems such as the ingredients of the MRF, magnetic field and sealing problems has being solved one by one.

The MRC. MRC uses magneto-rheological effect to replace traditional friction to transmit torque. When the current is switched on, the magnetic field is created in the clutch .The magnetic particles in the MRF begin to form chain structures and connect the two cylinder by shearing.

In this shear mode, there is relative motion between the cylinders, this movement makes the MRF being the shear state, and the shear stress could be changed by changing the magnetic flux density. As shown in figure 2. The transmission capacity is related with the magnetic field strength .When the magnetic saturation is reached, no more chains will be formed, and the clutch transmits the maximum torque.

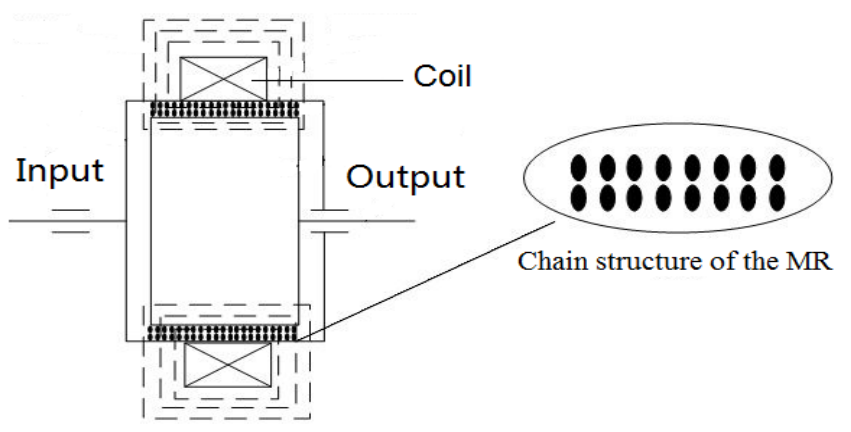

Fig. 2 The principle of MRC

The control of the clutch is easy. By controlling the current in the coil, the magnetic flux density is controlled, and the separation and combination of the MRC are realized.

\section{The Dual Clutch Transmission}

DCT is a new type of automobile transmission. It was developed based on MT-the parallel axis mechanical manual transmission [7]. The main feature of DCT is there has no power interruption during shifting. The vehicle's speed is stability and there has no impact caused by the shift. DCT brings a comfortable and safe experience for the drivers. One kind of DCT is shown in figure 3.

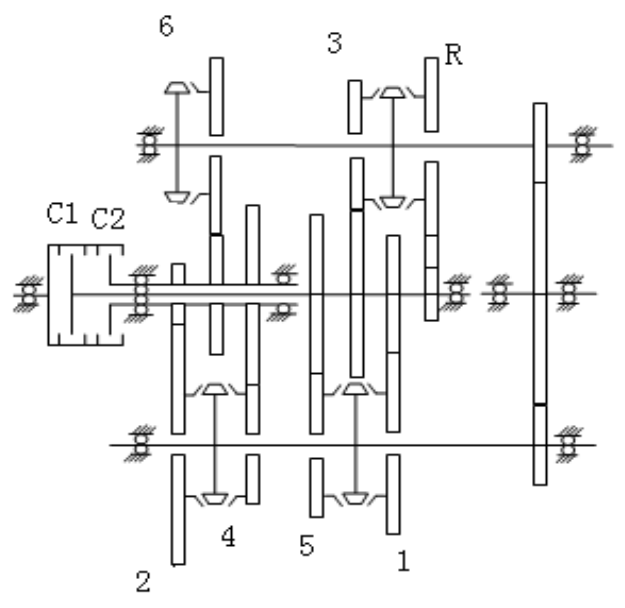

Fig. 3 The dual clutch transmission 
The input shaft is connected to the engine. The driven parts of the two clutches are connected to the two input shafts of the gear box respectively. Through the combination and separation of the two clutches during the driving process alternately, the power shift is realized. The shift is effectively and smooth.

\section{The MRDCT}

Based on the magneto-rheological effect of MRF and work principle of the DCT, designed MRDCT. This new type of DCT has simple structure, small abrasion, good controllability, and response rapidly [8]. The gear box in the two transmissions is the same. The difference between the MRDCT and traditional DCT is the dual clutch-MRDC, which is the key part of MRDCT.

The principle of MRDC. MRDC is a new kind of MRC; it is used to replace the traditional dual clutch. The principle of MRDC is shown in figure 4.The clutch includes the drive shaft, the input cylinders, the output cylinders, coils, MRF, bearings, seals, etc. The two clutches are multi-cylindrical, which can transmit a larger torque. There are many gaps in each clutch, where filled with MRF. Use seals to prevent the leakage. Two coils are placed on the side of the clutch, and the control modes are independent of each other.

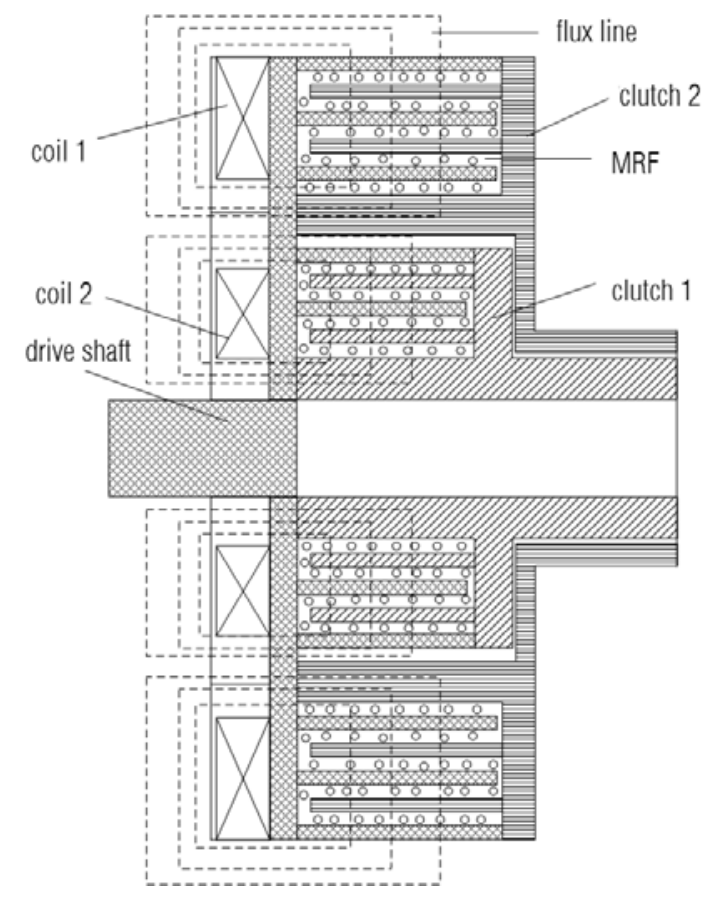

Fig. 4 The principle of MRDC

One coil controls one clutch. By changing the current in the coil, the magnetic flux density of the work areas can be changed. The clutch transmit different torque under different intensity, through that the clutch's separation, sliding and combination are controlled.

The torque calculation formula. For the MRDC, single clutch is required to transmit the target torque independently.

If the MRDC only has a cylinder, the torque of each clutch is as follows:

$$
T=\frac{4 \pi L r_{1}^{2} r_{2}^{2} \ln \left(r_{2} / r_{1}\right)}{r_{2}^{2}-r_{1}^{2}} \tau_{B}+\frac{4 \pi L r_{1}^{2} r_{2}^{2}\left(\omega_{2}-\omega_{1}\right)}{r_{2}^{2}-r_{1}^{2}} \eta
$$

Where: $L$ is the actual working length, $m ; r_{1}, r_{2}$ are the working radius, $m ; \omega_{1}, \omega_{2}$ are the angular velocity, $\mathrm{rad} / \mathrm{s} ; \tau_{B}$ is the shear yield stress, $\mathrm{Pa} ; \eta$ is the liquid viscosity, $\mathrm{Pa} \cdot \mathrm{s}$.

When the clutch is multi-cylindrical, there are $n$ work areas. The actual working length of the clutches is $L_{1}, L_{2}$, and the number of work areas is $n_{1}, n_{2}$, as shown in figure 5 . 
The torque in clutch 1 is:

$$
T_{1}=\sum_{1}^{n_{1}} \frac{4 \pi L_{1} r_{i 1}{ }^{2} r_{i 2}{ }^{2} \ln \left(r_{i 2} / r_{i 1}\right)}{r_{i 2}{ }^{2}-r_{i 1}{ }^{2}} \tau_{B i}+\sum_{1}^{n_{1}} \frac{4 \pi L_{1} r_{i 1}{ }^{2} r_{i 2}{ }^{2}\left(\omega_{i 2}-\omega_{i 1}\right)}{r_{i 2}{ }^{2}-r_{i 1}{ }^{2}} \eta
$$

The torque in clutch 2 is:

$$
T_{2}=\sum_{1}^{n_{2}} \frac{4 \pi L_{2} r_{i 1}{ }^{2} r_{i 2}{ }^{2} \ln \left(r_{i 2} / r_{i 1}\right)}{r_{i 2}{ }^{2}-r_{i 1}{ }^{2}} \tau_{B i}+\sum_{1}^{n_{2}} \frac{4 \pi L_{2} r_{i 1}{ }^{2} r_{i 2}{ }^{2}\left(\omega_{i 2}-\omega_{i 1}\right)}{r_{i 2}{ }^{2}-r_{i 1}{ }^{2}} \eta
$$

\section{The Magnetic Field Simulation}

Build a finite element analysis model in Ansoft Maxwell, as shown in figure 5. Set up the properties of the material of different parts. Use air gap to separate the two clutches. Simulate the distribution of the flux lines when the clutches are working separately.

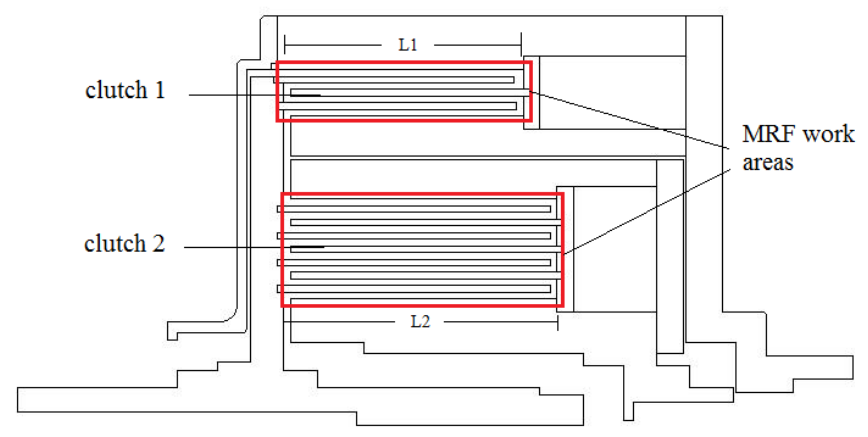

Fig. 5 The finite element analysis model

The distribution of the flux lines created by clutch 1 is shown in figure 6 . The current in clutch 1 is 3A, while $0 \mathrm{~A}$ in clutch2. We can see from figure 6: Most of the flux lines formed a closed loop in clutch 1 and the shell of clutch 2. And they pass through the MRF work areas of clutch 1 vertically and uniformly. Only few (less than 10\%) pass through the MRF work areas of clutch 2, and the strength is very small and can be neglected.
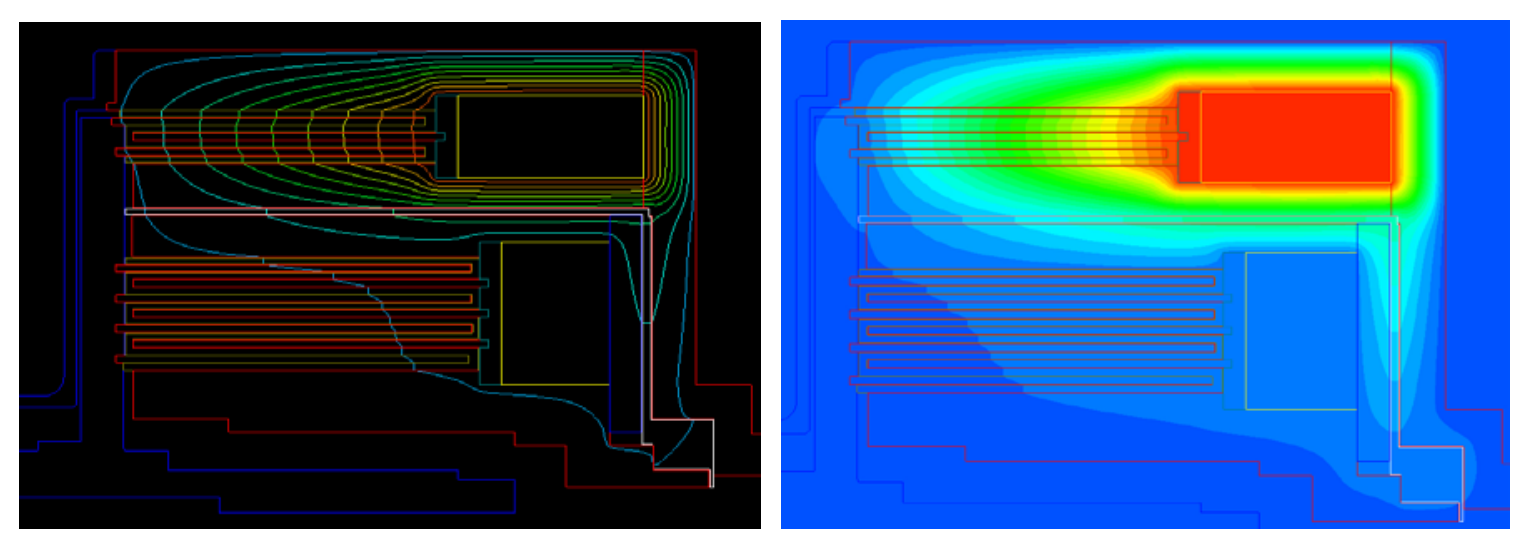

Fig. 6 The distribution of the flux lines created by clutch 1

The distribution of the flux lines created by clutch 2 is shown in figure 7 . The current in clutch 2 is $3 \mathrm{~A}$ and in clutch is $0 \mathrm{~A}$. Most of the flux lines formed a closed loop in clutch 2 and the shell of clutch 1 . 

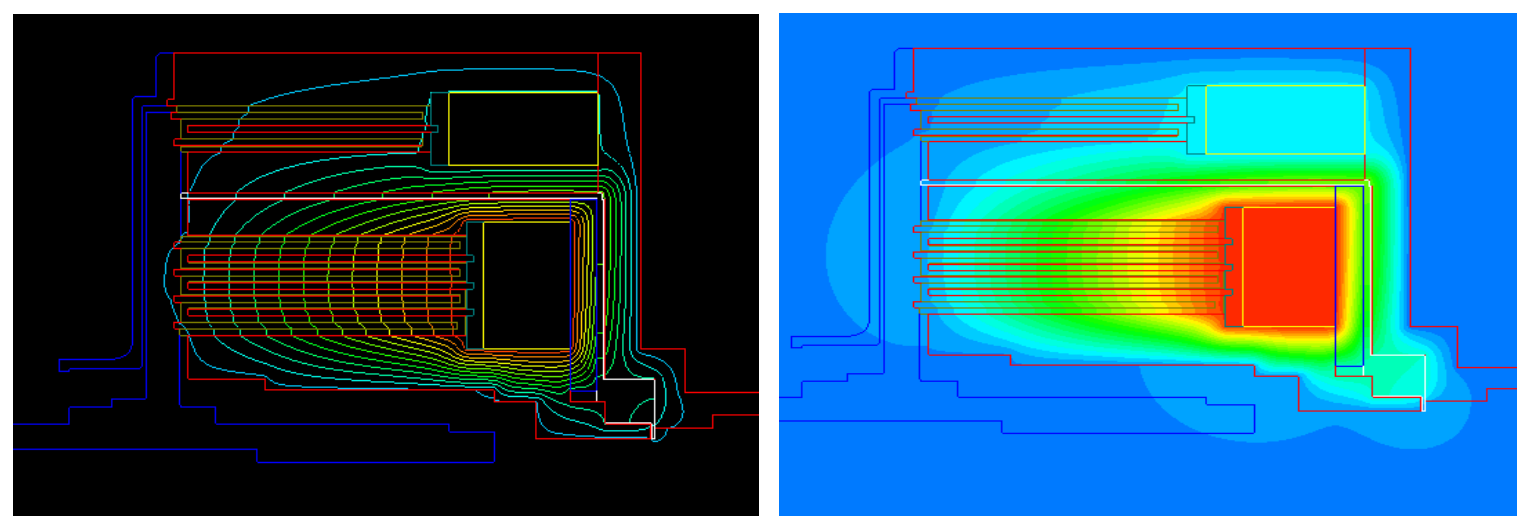

Fig 7 The distribution of the flux lines created by clutch 2

\section{Summary}

In this paper we introduced the MRF, the principle of MRC and DCT at first. Then put forward a new type of automobile transmission: MRDCT. MRDC is the key part of MRDCT, its principle was presented and torque transmission formula was expressed. Simulate the magnetic field of MRDC in Ansoft. The simulation results show that the magnetic circuits can be controlled by using proper magnetic materials and non-magnetic materials. The magnetic separation between the two single clutches is well and there has no electromagnetic interference. The principle of MRDC is feasible and can be used on automobile.

\section{References}

[1] Wang H, Yang Z and Han S J. Magneto-rheological Fluid Technology and Its Application[J].. Mechanical Engineering \& Automation, 2010.

[2] Fu L, Cheng T. Magneto-rheological Fluid and its Application in Engineering[J]. Journal of Shenyang Aerospace University, 2011.

[3] Alghamdi A A, Lostado R and Olabi A G. Magneto-Rheological Fluid Technology[J]. Materials Forming Machining \& Tribology, 2014, volume 252(1-3):224-228(5).

[4] Magnetic fluid clutch, Technical News Bulletin, National Bureau of Standards, 32/4 (1948), Page 54-60.

[5] Lee U, Kim D, Hur N, et al. Design Analysis and Experimental Evaluation of an MR Fluid Clutch[J]. Journal of Intelligent Material Systems \& Structures, 1999, 10(8):701-707.

[6] Joško Deur, Senior Member. Modeling of Electromagnetic Circuit of a Magnetorheological Fluid [C]. 2009 IEEE Multi-conference, Saint Petersburg, Russia. 2009, 113-118.

[7] Liu Z. Analysis of Double Clutch Automatic Transmission for Vehicles[J]. Transactions of the Chinese Society of Agricultural Machinery, 2005.

[8] Demin Chen, Jinhao Xu, Jingwen Pan et al. Research and Prospect of Automobile Magnetorheological Fluid Dual Clutch Transmission [C]. EMEIT-2012. 2012: 98-102. 